\title{
Natural Language Processing based Soft Computing Techniques
}

\author{
Jabar H. Yousif \\ Faculty of Computing and \\ Information Technology \\ Sohar University, P.O.Box.44, \\ P.C.311, Sohar, Sultanate of Oman
}

\begin{abstract}
This paper presents the implementation of soft computing (SC) techniques in the field of natural language processing. An attempt is made to design and implement an automatic tagger that extract a free text and then tag it. The part of speech taggers (POS) is the process of categorization words based on their meaning, functions and types (noun, verb, adjective, etc). Two stages tagging system based MPL, FRNN and SVM are implemented and designed. The system helps to classify words and assign the correct POS for each of them. The taggers are tested using two different languages (Arabic and Hindi). The Word disambiguation issue has been solved successfully for Arabic text. Experience has shown that the proposed taggers achieved a great accuracy (99\%).
\end{abstract}

\section{General Terms}

Computer Science, NLP, Soft Computing,

Information Systems, Evolutionary Computing.

\section{Keywords}

Artificial Intelligence, Artificial Neural Networks, Neural

Tagger, Part of Speech, Optimizing Techniques.

\section{INTRODUCTION}

Soft Computing (SC) refers to a collection of computational paradigms which attempt to utilize tolerance for imprecision, uncertainty, robustness and tiny solution cost to formularize real-world problems. SC generally includes Artificial Neural Networks (ANN), Fuzzy Logic (FL), Evolutionary Computing, Genetic Algorithms (GA) and Rough Set Theory [30]. The Main characteristics of SC are their ability to evaluate, decide, check, and calculate within a vague and imprecise domain, emulating the human abilities in the execution to learn from past experience. Natural Language Processing (NLP) can be defined as an automatic or semiautomatic approach of processing the human language [17, 25]. Recently, the application of Arabic and Hindi languages processing has become a primary focus of research and commercial development. Most of NLP application often includes speed and accurate POS tagger as one of its main core components [21]. The Part of Speech (POS) is a classification of words according to their meanings and functions. The POS tagger plays a crucial and important role for most of the NLP applications such as machine translation, information extraction, speech recognition, as well as grammar and spelling checkers [13]. Moreover, the accuracy of the POS tagging is determined by factors like ambiguous words, phrases, unknown words and multipart words. There are specific features that excite scientist to espouse neural network based solution in solving problems [12, 15]. The most important features are massive parallelism, uniformity, generalization ability, distribution representation and computation, learn-ability, trainability and adaptation. Neural approaches have been performed successfully in many aspects of artificial intelligence such as image processing, NLP, speech recognition, pattern recognition and classification tasks [2]. The Recurrent Neural Networks (RNN) is a network of neurons with feedback connections, which are biologically more plausible and computationally more powerful than other adaptive models like Hidden Markov Models (HMM), FeedForward Networks and Support Vector Machines (SVM) [14,16 and 24]. The SVMs are considered as supervised learning method that used to perform binary classification and regression tasks. They belong to a family of generalized linear classifiers. The main advantages of SVM are that they simultaneously minimize the experimental classification error and maximize the geometric margin $[6,12]$.

\section{THE DIFFERENCES BETWEEN POS TAGGER MODELS}

The Part-of-speech tagging is complicated process not just having a list of words and their parts of speech as at times, some words can represent more than one part of speech, and some can be in form of ambiguous phrases [4, 5]. Hence, for a large training data, it is hard to build a POS tagger that can tag with an accuracy of 100 percent. Typically, deferent approaches have been implemented to address the part of speech tagger such as the rule-based [9, 18 and 19], stochastic [7, 10 and 11], neural network $[1,12,13,14,15,16,20,22$, 23 and 26] or the hybrid systems [8]. The rule based and stochastic approaches need a vast amount of data in order to adapt and implement the POS tagger. It has been known that the neural approaches only use a little amount of data to perform the training and learning stages. Moreover, the neural-based approaches not only consummate the associations (word-to-tag mappings) from a representative training data set but they can also be generalized to the unseen $[1,17]$. Overall, several advantages of the stochastic taggers can be identified over the rule-based taggers as they avoid the need for diligent manual rule building and probably obtain the useful information that may not be noticed by humans. However, these probabilistically driven ones have the disadvantage in which the linguistic information is only captured indirectly, in large tables of statistics. In contrast, the rule-based taggers need the minimum storage requirements and at the same time, are more portable [17]. 


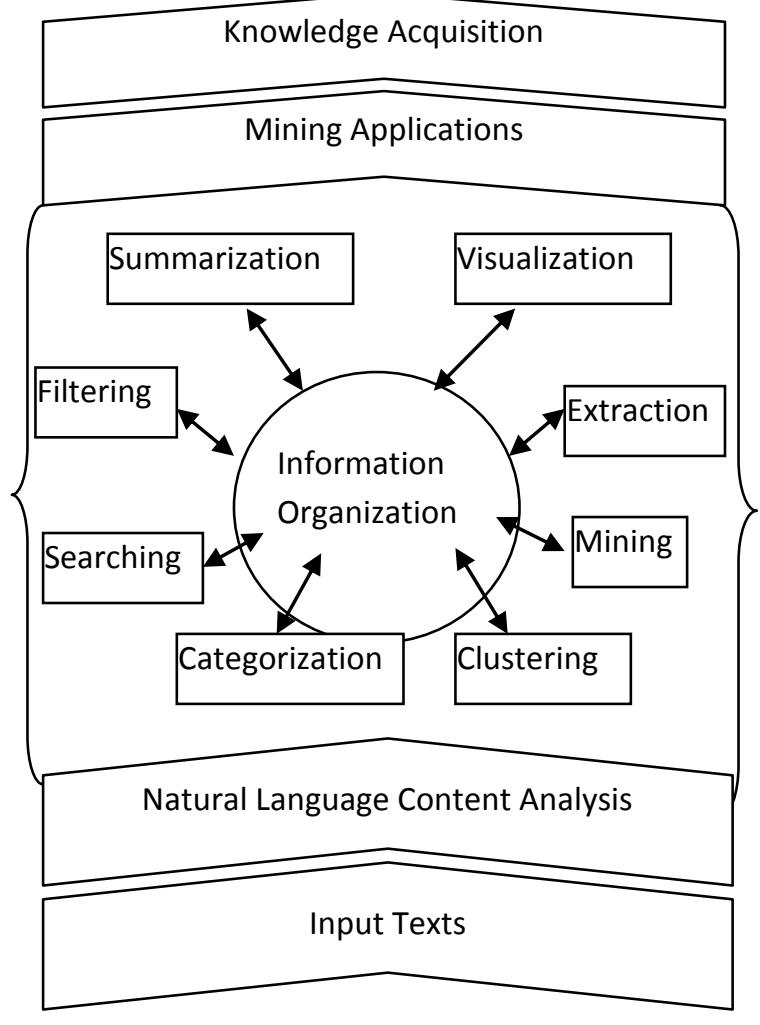

Figure1 : NLP process nad its applications

\section{PERFORMANCE MEASURES}

The performance evaluation of classification process is a crucial matter in the machine learning systems, because it is unfeasible to contrast learning algorithms or even know whether a hypothesis should be used. The most important attribute in the assessment of a part-of-speech tagger is accuracy [28]. Thus, the quality of the output depends on the comparability of conditions [17] such as: **Tag-set size: Normally, using a small number of tag-set can help to give high accurate tagging but it does not offer as much information or disambiguation between the lemmas as a larger one would.

**The corpus type: A corpus (corpora is the plural) is a set of text that collected for a purpose. The type of corpus affects the quality of taggers output when the genre or type of the corpus data differs from the tagged material.

**Vocabulary type: the tagging of specific texts such as the medical or legal texts requires a training corpus that has examples of such texts; otherwise the unknown words will be unnaturally high). Likewise, the high instance of idiomatic expressions in the literary texts often leads to inaccuracy.

However, the ambiguous words and phrases, unknown words and multi-part words can affect the accuracy of POS tagging. Ambiguity appears at different levels of the language processing sequence such as syntax or semantic phase [28].

\section{RELATED WORK}

This section is presented a survey of previous work in fields of part of speech tagger using of rule based, stochastic and neural networks. Schmid [26] established a Net-Tagger which trained on a large corpus called Penn Treebank. This tagger has a context window of three preceding words and two succeeding words The corpus performed significantly and compared to statistical approaches based on "Trigram model" and "Hidden Markov model" (HMM). Diab et al. [10] they utilized a SVM approach toward automatically tagging POS and annotate base phrases (BPs) in Arabic text. She attained score of 99.12 for tokenizing when $\mathrm{F}_{\beta}=1$, and score of tagging accuracy is $95.49 \%$. While, recorded score of 92.08 for chunking when $F_{\beta}=1$. Pérez-Ortiz [23] implemented a Discrete-time Recurrent Neural Networks (DTRNN) tagger to tag ambiguous words from the sequential information stored in the network's state. The experiments computed the error rates when tagging text taken from the Penn Treebank corpus. Ahmed [1] used MLP-tagger with three-layers using error back-propagation learning algorithm. The tagger was implemented on SUSANNE English tagged-corpus consisting of 156,622 words. The MLP-tagger is trained using $85 \%$ of the corpus. Based on the tag mappings learned, the MLPtagger demonstrated an accuracy of $90.04 \%$ on test data that also included words unseen during the training. Jabar [23] implemented an Arabic part of speech based multilayered perceptron. The experiments evinced that the MLP tagger has high accurate (of 98\%), with low training time and fast words tagging. they used a little amount of data to achieve the adaptation and learning of network. Jabar [12] proposed Arabic part of speech based support vectors machine. The radial basis function is used as a linear function approximation. The experiments evinced that the SVM tagger has a high accuracy and recall about (99.99\%). Jabar [14] proposed an Arabic part of speech based Fully Recurrent Neural Networks (FRNN). The back-propagation through time (BPTT) learning algorithm is used to adjust the weight of the network and associate inputs to cyclic outputs. In order to accurately predict the syntactic classification tagging, an encoding criteria is also presented and performed. The experiments evinced that the FRNN tagger is accurate and achieved $94 \%$ in classification phase. Similarly, the POS disambiguation problem was successfully solved. Khoja [19] proposed the APT Arabic Part-of-Speech Tagger which used a combination of both the statistical and rule-based techniques. The APT tag-set are derived from the BNC English tag-set, which was modified with some concepts from the traditional Arabic grammar.

\section{TAGGERS DESIGN}

The proposed system is consisting of two main stages as depicted in Figure 2. The key function of first stage is to prepare the input data sets for next stage. This stage is written and implemented using VBA commands for Excel. While, the main function of second stage is to implement the automatic taggers. These taggers are designed and implemented using NeuroSolutions for Excel software. The first stage is called "pre-processing phases"[17]. It is implemented and utilized to achieve the following tasks: Text Normalization, Text Tokenization and Text Encoding.

The Text normalization is used to convert the input text from free text into suitable forms to be used in next stage. In general, the input text can be configured either into a text file or XML file. Therefore, the system is designed to disregard all the HTML tags and extract the pure contents of the document. Then, the text tokenization is distributed the pure text into simple tokens such as numbers, punctuation, symbols, and words. An algorithm has been developed to implement and perform the text tokenization task. Lastly, the text encoding is performed to transform the input data into a suitable digital form, which the network can identify and use $[13,17]$.

The proposed encoding method aims to solve the drawbacks of previous encoding schemes. Consequently, it aims to increase the number of significant positions and decrease the usage of memory storage. 
Moreover, the proposed scheme uses new concepts like the probability of the word and the numerical values of each word. An algorithm is developed to compute the probability of each word. This algorithm is written and implemented using VBA commands for Excel. Finally, the numerical value of each word in the input based on the new encoding scheme is determined. Subsequently, every word in the sentence is associated with a bit-vector, i.e., the size of which is equal to the number of different grammatical categories (for parts of speech) in a specific language.

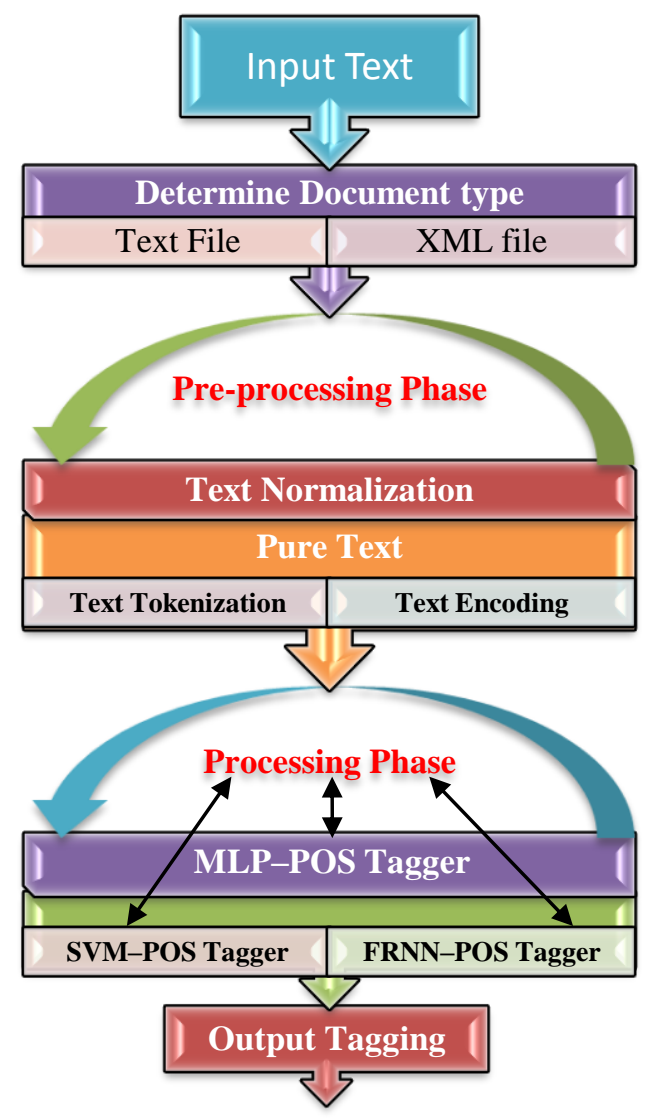

Figure 2: System Architecture

The second stage is "processing phase" which is used to design and implement automatic taggers which include the following tasks:

**The MLP tagger [13, 15 and 16]: This task is designed to implement the POS tagger for Arabic text using the Multilayered Perceptron technique. The architecture of the network has one hidden layer, 23 processing elements tagged as input, and 23 processing elements tagged as output. The maximum number of epochs is 1000 . The TanhAxon is implemented as a transfer function in the hidden and output layer. The TanhAxon applies a bias and Tanh function to each neuron in the layer.

**The Recurrent tagger [14]: This task is designed to implement the POS tagger for Arabic text using the fully Recurrent Neural Networks technique. The tagger has one hidden Fully Synapse (The Synapse family implements the dynamic linear mapping characteristics of the neuron. A synapse connects two layers of axons) with 230 processing elements as input and 23 processing elements as output. A static controller for a network with multi-channels tapped delay line memory structure (TDNNAxon) which has 10 tapes, usually used as the Input Axon. Likewise, in both the hidden and output layers, the TanhAxon as a transfer function is implemented. The RNN has 23 processing elements (Columns) tagged as Input and 23 processing elements (Rows) tagged as Output.

**The SVM tagger [12]: This task is used to implement the POS tagger for Arabic text using the terminology of super vector machine algorithms. The SVM architecture have 23 PEs as input set xi , 23 PEs as output set di and have no hidden layer. The maximum number of epochs is 1000 and set the step size to 0.01 . The learning algorithm is based on the Adatron algorithm which is extended to the RBF network.

\section{EXPERIMENTS AND RESULTS}

The experiments undertaken are achieved using the Arabic tag-set which is proposed by Khoja [19]. The tag-set contains 177 tags that include various categories. The extraction of words into basic roots is not considered in this study. This study supposed that the words were segmented before POS tagging began. The experiments covered the three proposed taggers in this paper SVM tagger, MLP tagger and FRNN tagger. The input text is encoded into a suitable form and then it is divided into three categories; training data sets, cross validation data sets and test data sets .The Cross validation computes the error in a test data sets at the same time that the network is being trained with the training set. The Genetic Algorithm (GA) is used as a heuristic optimization in the problem of finding the best network parameters [27]. It establishes with an initial population of randomly created bit strings.

These initial samples are encoded and applied to the problem. The study under taken is used the GA methods for improving the learning rule parameters such as step size and momentum value [13, 14 and 17]. This will enable the optimization of the momentum values for all Gradient components in NeuroSolutions software that use momentum. Besides, it used to determine the number of processing elements. Likewise, to tolerate the enhanced fit specimen in the population to reproduce at a higher rate is to use a selection method based on the roulette wheel selection technique. The standard method to assess the tagger performance is usually determined by the percentage of correct tag assignments.

The NeuroSolutions for Excel software is provided six methods to test the networks performance such as the mean squared error (MSE), the normalized mean squared error (NMSE), the correlation coefficient(r), the percent error, Akaike's information criterion (AIC) and Rissanen's minimum description length (MDL) criterion [17].

The NMSE is the estimation of the overall deviations between predicted values and measured by the network. It is defined as follows:

$$
\text { NMSE }=\frac{\sum_{j=0}^{P} \sum_{i=0}^{N}\left(d_{i j}-y i_{j}\right)^{2}}{\sum_{j=0}^{P} \frac{N \sum_{i=0}^{N} d_{i j}{ }^{2}-\left(\sum_{i=0}^{N} d_{i j}\right)^{2}}{N}}
$$

The MSE "mean squared error" is two times the average cost which is computed as follows:

$$
M S E=\frac{\sum_{j=0}^{P} \sum_{i=0}^{N}\left(d_{i j}-y i_{j}\right)^{2}}{N P} .
$$

Where, $\mathrm{P}$ is the number of output processing elements. 
$\mathrm{N}$ is the number of exemplars in the data set.

$\mathrm{yi}_{\mathrm{j}}$ is the network output for the exemplar $\mathrm{i}$ at processing element $\mathrm{j}$.

$\mathrm{d}_{\mathrm{ij}}$ is the desired output for the exemplar $\mathrm{i}$ at processing element $\mathrm{j}$.

The correlation coefficient $(r)$ is the rate relation between a network output $\mathrm{x}$ and a desired output $\mathrm{d}$. It is defined as follows:

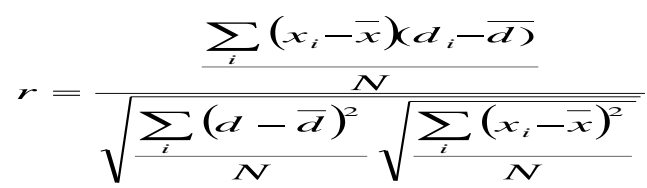

Usually, the MSE "mean squared error" is used as evaluation function for the network output reliability. The best network results for training data of the proposed taggers (MLP, FRNN and SVM) reported a minimum and final mean squared error (MSE) as depicted in Tables 1,2 and 3 accordingly. Moreover, Figures 4, 5, and 6 are illustrated the training results graph of proposed neural taggers MLP, FRNN and SVM respectively. Figure3 shows the comparison results of MSE of Proposed taggers. The MLP network recorded a final MSE of 0.000103638. And the FRNN network recorded a final MSE of 0.020686609. Lastly the SVM network recorded a final MSE of 0.000878348 .

Table 1. MSE for MLP tagger

\begin{tabular}{|l|c|c|}
\hline Best MLP Networks & Training & Cross Validation \\
\hline Run NO. & 2 & 2 \\
\hline Epoch NO. & 1000 & 1000 \\
\hline Minimum MSE & 0.000103638 & 0.000104718 \\
\hline Final MSE & 0.000103638 & 0.000104718 \\
\hline
\end{tabular}

Table 2. MSE for FRNN tagger

\begin{tabular}{|l|c|c|}
\hline Best FRNN Networks & Training & Cross Validation \\
\hline Run NO. & 3 & 3 \\
\hline Epoch NO. & 1000 & 1000 \\
\hline Minimum MSE & 0.020686609 & 0.02351733 \\
\hline Final MSE & 0.020686609 & 0.02351733 \\
\hline
\end{tabular}

Table 3. MSE for SVM tagger

\begin{tabular}{|l|c|c|}
\hline Best SVM Networks & Training & Cross \\
\hline Run NO. & 1 & 1 \\
\hline Epoch NO. & 1000 & 1000 \\
\hline Minimum MSE & 0.000878348 & 0.008483563 \\
\hline Final MSE & 0.000878348 & 0.008483563 \\
\hline
\end{tabular}

\section{COMPARISON \& CONCLUSIONS}

\subsection{Comparison with related work}

The comparison study has to be implemented carefully because the features used here to identify the languages and the tag sets are different with the previous studies [12]. On the other hand, the comparison of proposed taggers with other existing taggers is difficult matter, because the tagger accuracy relies on numerous parameters such as language difficulty (ambiguous words, ambiguous phrases), the language nature (English, Arabic, Hindi, Chinese, etc), the training data size, the tag-set size and the evaluation measurement criteria [13, 14]. The Tag-set size has a great impact on the tagging process. The proposed taggers are assessed using the measurement of Accuracy, besides MSE aspects. In addition, the amount of data used in the training and learning stages is considered. In comparison study of proposed taggers with the results of other taggers explained that the proposed taggers achieved a high accuracy rate when using GA optimization techniques which improved the values of the momentum rate and the step size. The proposed taggers (SVM, MLP and FRNN) achieved high accuracy of $99 \%$ at last experiments when the GA optimization process is implemented. Table4 summarizes the comparison information with other researchers. Figure7 illustrates the overall comparison results.

\subsection{Conclusions}

The research mainly aims to implement an automatic and accurate tagging system which can be used as a main core component for NLP applications. The automatic part of speech tagging system is implemented based on neural network techniques, which has the ability to tag the free texts automatically. The study demonstrated variant kinds of taggers which can solve the problem associate with the contraction of languages such as Arabic part of speech and Hindi part of speech. The new approaches are highly accurate with low processing time and high speed tagging. Two stages automatic tagging system based SVM, MPL and FRNN are implemented and designed. The proposed system helps to classify words and assign the correct POS for each of them. The results are greatly encouraging, with correct assignments and recall about 99\%. The genetic Algorithm is used to optimize the network variables like the momentum rate and step size. The words disambiguation is solved in Arabic POS taggers.

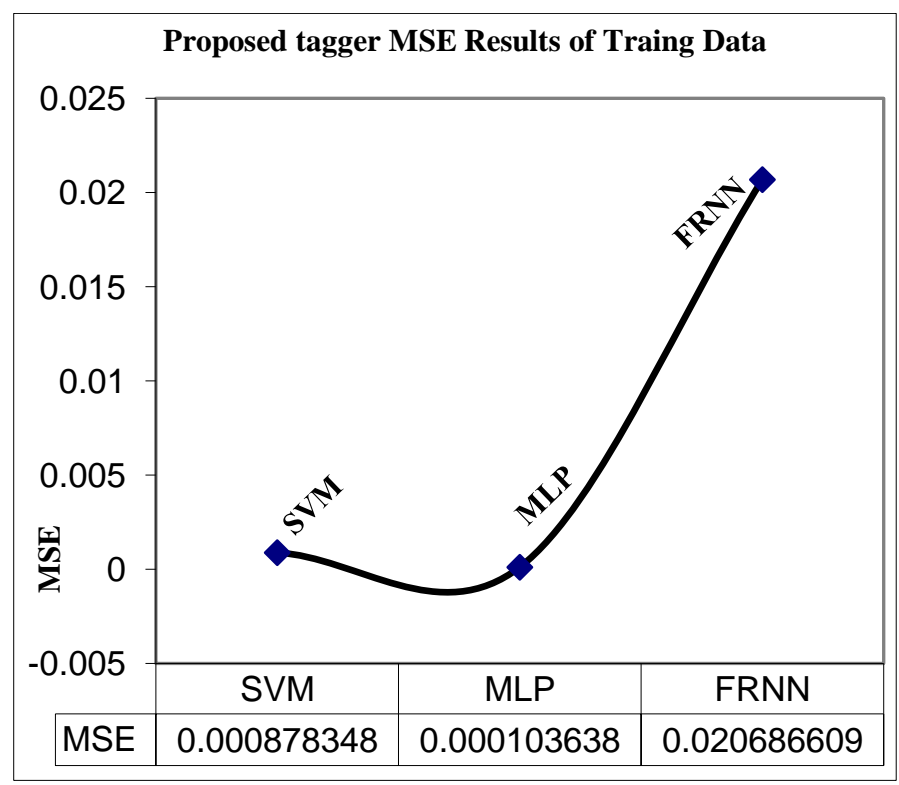

Figure3: MSE of Proposed taggers 


\section{FUTURE WORK}

This paper presented the design and implementation of automatic tagger that can tag a free text directly and combining each word with their correct part of speech. The work focuses only on two types of files (text file and HTML files). Therefore, it is preferable to include more files type. And, if it is possible to extract the text directly from the website, it will be very encouraging. The current work comprises of two separate stages, first pre-processing phase which implemented using VBA codes. Besides, the second stage is processing phase which is implemented using the NeuroSolutions software. In order to produce a portable system which it can be used with any other applications, it is very useful that the phases are merged into one part.

\section{REFERENCES}

[1] Ahmed. "Application of Multilayer Perceptron Network for Tagging Parts-of-Speech", Proceedings of the Language Engineering Conference (LEC'02), IEEE, 2002.

[2] Aleksander, Igor, and Morton, Helen, An Introduction to Neural Computing, Chapman and Hall, London, 1990.

[3] Al-Sulaiti's Latifa. "Online corpus". http://www.comp.leeds.ac.uk/latifa/research.htm.

[4] Attia, M. " A large-scale computational processor of the Arabic morphology and applications", MSc. thesis, Dept. of Computer Engineering, faculty of Engineering, Cairo University, 2000.

[5] Beesley, K. "Finite-State Morphological Analysis and Generation of Arabic at Xerox Research: Status and Plans", ACL, Arabic NLP Workshop, Toulouse, 2001.

[6] Ben-Hur, A., Horn, D., Siegelmann, H. T., and Vapnik, V. (2001),Support vector clustering, Journal of Machine Learning Research, 2:125-137.

[7] Brants, T. "TnT- a statistical part-of-speech tagger", proceedings of the $6^{\text {th }}$ ANLP conference, Seattle, WA, 2000

[8] Brill, E. "Unsupervised learning of disambiguation rules for part of speech tagging". Proceedings of third ACL Workshop on Very Large Corpora, 1995.

[9] Brill, E. "A simple rule-based part-of-speech tagger", proceedings of ANLP-92, $3^{\text {rd }}$ Conference on Applied Natural Language Processing, pp 152-155, Trento, IT, 1992.

[10] Diab, M., Kadri H. \& Daniel J. "Automatic tagging of Arabic text: from raw text to base phrase chunks", proceedings of HLT-NAACL-04, 2004.

[11] Gimenez, J. \& Llu'is M. "Fast and accurate part-ofspeech tagging: The SVM approach revisited", proceedingsof the International conference on recent advances on natural language processing, Borovets, Bulgaria, 2003.

[12] Jabar H. Yousif, \& Sembok, T., "Arabic Part-Of-Speech Tagger Based Support Vectors Machines", proceedings of International Symposium on Information Technology,Kuala Lumpur Convention Centre, ISBN 978-1-4244-2328-6@IEEE, Malaysia, August 26-29, pp: 2084-2090, 2008. URL:http://ieeexplore.ieee.org/xpl/freeabs_all.jsp?arnum ber $=4632066$.
[13] Jabar H. Yousif, \& Sembok, T., "Design And Implement An Automatic Neural Tagger Based Arabic Language For NLP Applications", Asian Journal of Information Technology Vol. 5, Issue 7, ISSN 1682-3915, pp 784789, 2006. DOI: 10.3923/ajit.2006.784.789.

[14] Jabar H. Yousif, \& Sembok, T., "Recurrent Neural Approach Based Arabic Part-Of-Speech Tagging", proceedings of International Conference on Computer and Communication Engineering (ICCCE'06), Vol 2, ISBN 983-43090-1-5@ IEEE, KL-Malaysia, May 9-11, 2006.

[15] Jabar H. Yousif, \& Sembok, T., "Arabic Part-Of-Speech Tagger Based Neural Networks", proceedings of International Arab Conference on Information Technology ACIT2005, ISSN 1812/0857. JordanAmman-2005.

[16] Jabar H. Yousif, and Dinesh Kumar Saini ,"Hindi PartOf-Speech Tagger Based Neural Networks" , Journal of Computing, Volume 3, Issue 2, pp59-65 ISSN 21519617 ,NY, USA, February 2011.

[17] Jabar H. Yousif, "Information Technology Development", LAP LAMBERT Academic Publishing, Germany ISBN 9783844316704, 2011.

[18] 10Khoja S, Garside, R. \& Gerry, K. "An Arabic tagset for the morphosyntactic tagging of Arabic", corpus linguistics, Lancaster University, Lancaster, UK, 2001.

[19] Khoja, S. "APT: Arabic part-of-speech tagger", proceedings of the student workshop at the second meeting of the north American chapter of the association for computational linguistics (NAACL2001), Carnegie Mellon University, Pennsylvania, 2001.

[20] Ma, Q., Uchimoto, K., Murata,M. \& Isahara,H. "Elastic neural networks for part of speech tagging", proceedings of IJCNN'99, pp 2991-2996, Washington, DC, 1999.

[21] Mahtab, N. \& Choukri, K. "Survey on Industrial needs for Language Resources", 2005.Online "http://www.nemlar.org/Publications/Nemlar-report-indneeds_web.pdf".

[22] Marques, N. C. \& Gabriel. P. L. "Using neural nets for Portuguese part-of-speech tagging", proceedings of the $5^{\text {th }}$ international conference on the cognitive science of natural language processing, Dublin City University, Ireland, 1996

[23] 15Persz-ortz A. J. \& Forcada M. L. "Part-of-speech tagging with recurrent neural networks", proceedings of the International Joint Conference on Neural Networks, IJCNN- IEEE2001:1588-1592, 2001

[24] Principe, J.C. ,Euliano,N.R. \& Lefebvre, W.C. "Neural and adaptive systems, fundamentals through simulations", John Wiley \& Sons, NY, 2000.

[25] Rababaa, M., , Batiha, K., \& Jabar H. Yousif, "Towards Information Extraction System Based Arabic Language", International Journal of Soft Computing ,Vol.1, No.1, ISSN: $\quad 1816-9503 \quad$ PP 67-70, 2006 http://medwelljournals.com/abstract/?doi=ijscomp.2006. 67.70

[26] Schmid, H. "Part-of-speech tagging with neural networks", proceedings of COLING-94, Kyoto, Japan, pp 172-176, 1994. 
[27] Srinivas, M. \& Patnaik, L. M. Genetic algorithms: a survey", IEEE Computer 27(6), pp17-26, 1994.

[28] Ueffing, N. Macherey, K. \& Ney, H. 2003. Confidence measures for statistical machine translation. New Orleans: MTSummit.
[29] Weischedel, R., et al. "Coping with ambiguity and unknown words through probabilistic models", Computational Linguistics. 19(2), pp359-382, 1993.

[30] Ying Dai, Basabi Chakraborty and Minghui Shi. "Kansei Engineering and Soft Computing: Theory and Practice." IGI Global, 2011. 1-436. Web. 19 Mar. 2012 doi:10.4018/978-1-61692-797-4

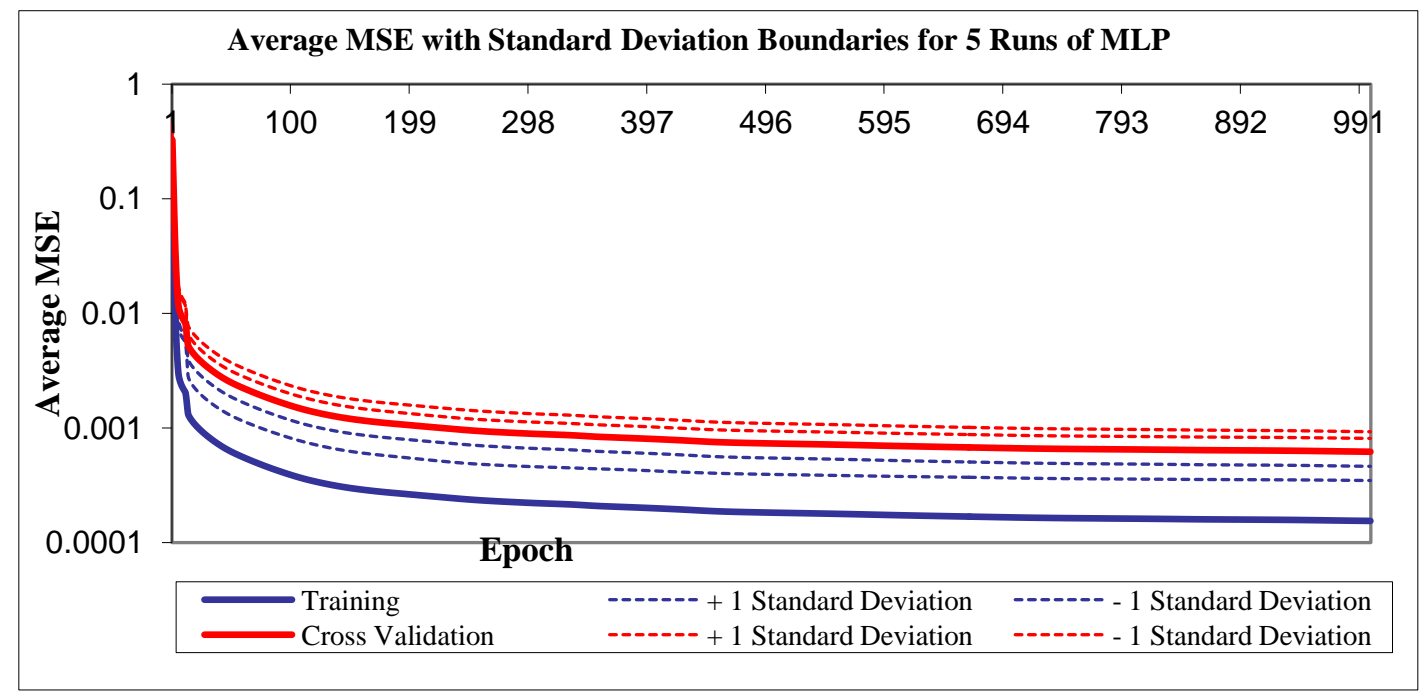

Figure 4: Average MSE with Standard Deviation Boundaries for 5 Runs of MLP

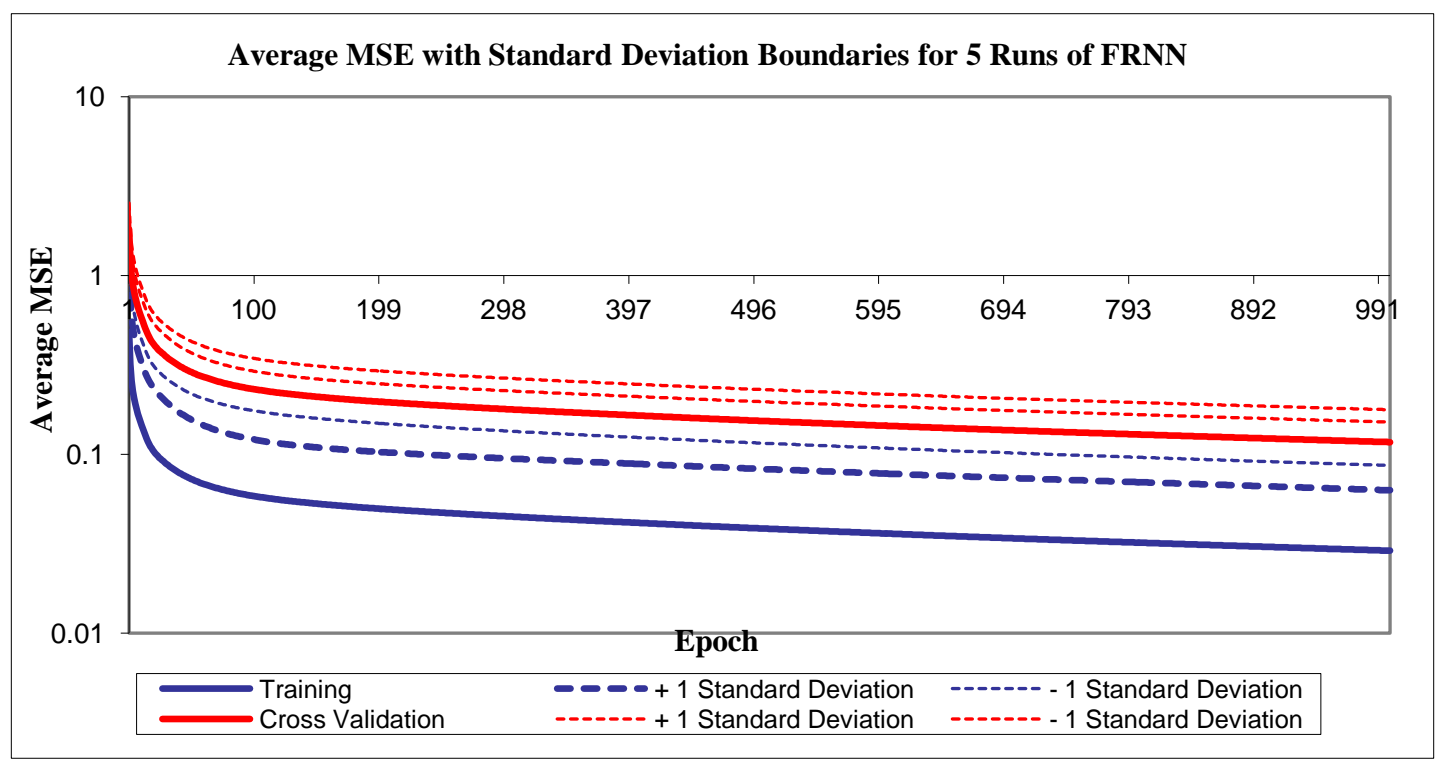

Figure 5: Average MSE with Standard Deviation Boundaries for 5 Runs of FRNN 


\begin{tabular}{|c|c|c|c|c|c|c|c|c|}
\hline & Schmid[17] & Pérez[15] & Ahmed[1] & Khoja[11] & Diab[8] & Jabar[12] & Jabar[13] & Jabar[14] \\
\hline Method Used & NN & DT-RNN & NN & Rule base & SVM & NN & NN & NN \\
\hline Tag-set type & English & English & English & Arabic & Arabic & Arabic & Arabic & Arabic \\
\hline Corpus size*10 & 45 & 0.465 & 0.015662 & 0.5 & $\begin{array}{c}0.04519 \\
\text { sentences }\end{array}$ & 0.5 & 0.5 & 0.5 \\
\hline Train Data percent & $44.4 \%$ & $100 \%$ & $85 \%$ & $100 \%$ & $80 \%$ & $10 \%$ & $10 \%$ & $10 \%$ \\
\hline NO. of Tag size & 48 & 19 & 48 & 131 & 19 & 131 & 131 & 131 \\
\hline Accuracy \% & 96.22 & 92 & 90.4 & 90 & 94.5 & 99 & 99 & 99 \\
\hline
\end{tabular}

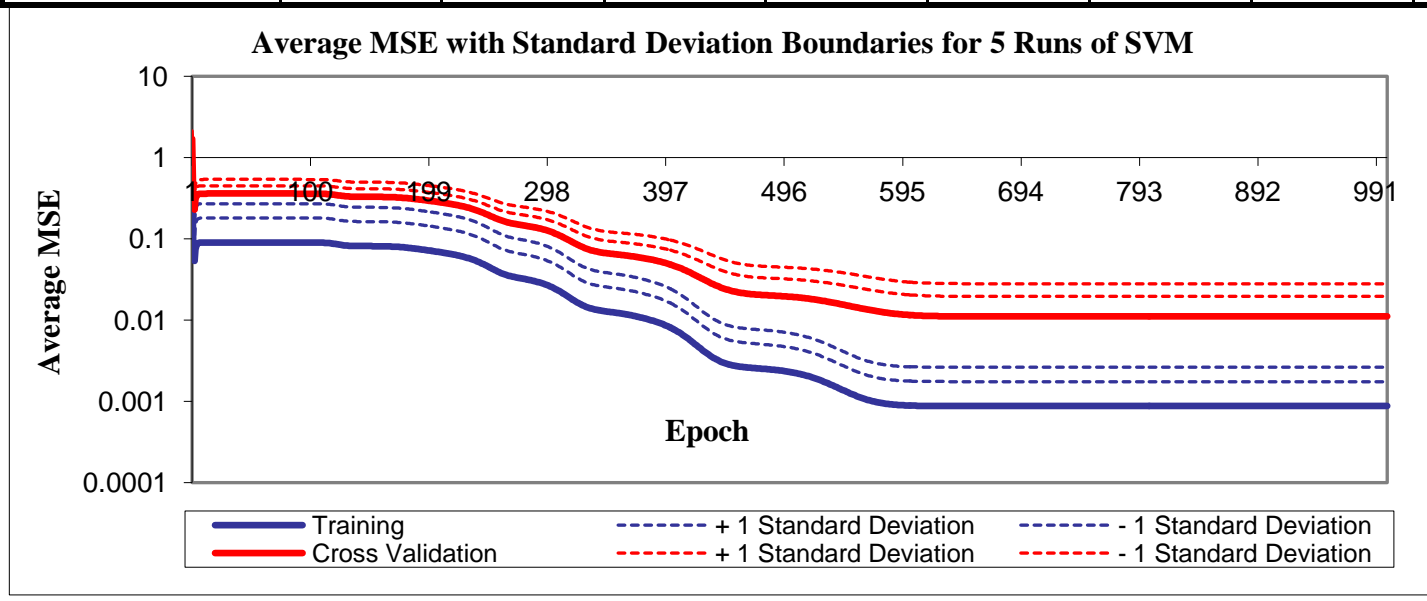

Figure 6: Average MSE with Standard Deviation Boundaries for 5 Runs of SVM

Table4: The comparison results of proposed taggers \& other taggers

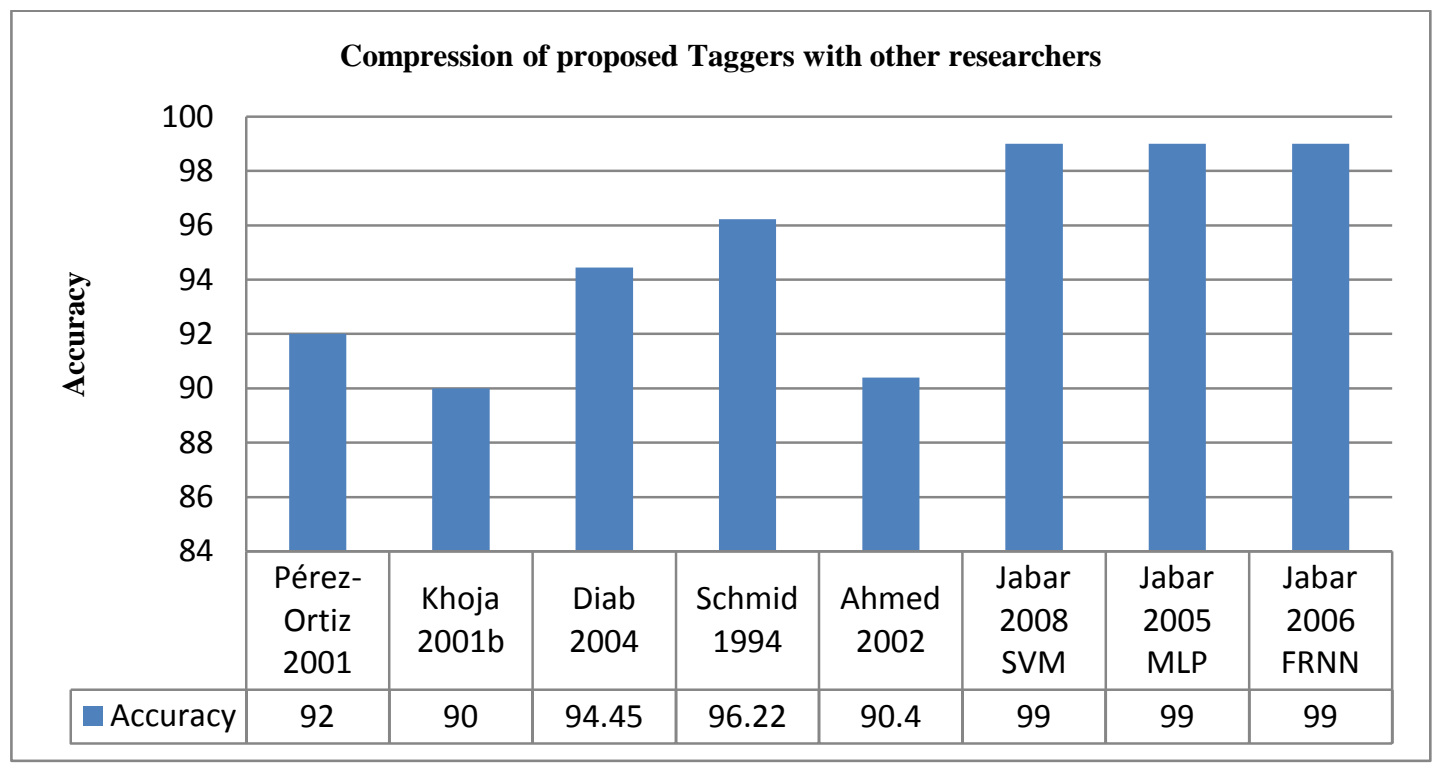

Figure7: the Compression of proposed Taggers with other researchers 